\title{
THE SEVENTEENTH SUMMER MEETING OF THE AMERICAN MATHEMATICAL SOCIETY.
}

THE seventeenth summer meeting of the Society was held at Columbia University on Tuesday and Wednesday, September 6-7, 1910, two sessions being arranged for each day. The attendance included the following thirty-six members :

Professor M. J. Babb, Professor G. D. Birkhoff, Professor J. W. Bradshaw, Professor E. W. Brown, Dr. H. T. Burgess, Mr. R. D. Carmichael, Professor G. G. Chambers, Professor A. B. Coble, Professor F. N. Cole, Dr. G. M. Conwell, Professor John Eiesland, Professor T. S. Fiske, Professor W. B. Fite, Professor O. E. Glenn, Professor C. C. Grove, Professor H. E. Hawkes, Dr. A. A. Himowich, Professor J. I. Hutchinson, Professor O. D. Kellogg, Professor C. J. Keyser, Professor P. A. Lambert, Dr. D. D. Leib, Mr. Joseph Lipke, President E. O. Lovett, Dr. H. H. Mitchell, Professor Arthur Ranum, Professor D. A. Rothrock, Dr. J. E. Rowe, Professor H. E. Slaught, Dr. Clara E. Smith, Professor Virgil Snyder, Professor R. P. Stephens, Dr. W. M. Strong, Professor Oswald Veblen, Professor H. S. White, Professor Alexander Ziwet.

Vice-President Professor Hutchinson occupied the chair. The Council announced the election of the following persons to membership in the Society: Mr. F. S. Bartlett, General Electric Company, Schenectady, N. Y.; Mr. R. D. Beetle, Dartmouth College; Professor N. C. Grimes, University of Arizona; Professor F. T. H'Doubler, Miami University; Mr. Robert Henderson, Equitable Life Assurance Society, New York, N. Y. ; Mr. G. F. McEwen, Stanford University ; Professor Josephine A. Robinson, Berea College. Ten applications for membership were received.

Professors P. F. Smith, Osgood, and E. H. Moore were appointed a committee to report to the Council at the October meeting a list of nominations for officers and other members of the Council to be elected at the annual meeting in December.

On Tuesday evening twenty-five members gathered at the usual dinner, which is always an agreeable incident of the meetings. 
The following papers were read at the summer meeting:

(1) Professor L. E. Dickson: "On the factorization of integral functions with $p$-adic coefficients."

(2) Professor L. E. Dickson : "Determination of the binary modular groups and their invariants."

(3) Professor O. E. GLenN : "On the structure of $p$-ary forms."

(4) Mr. R. D. Carmichael: "Linear difference equations and their analytic solutions."

(5) Dr. H. T. Burgess: "The simultaneous reduction of a quadratic and a bilinear form by the same transformation on both $x$ 's and $y$ 's."

(6) Professor A. B. Coble : "On the reduction of the sextic equation to the Valentiner form problem."

(7) Professor Virgil SNyder : "The involutorial birational transformation of the plane, of order 17."

(8) Professor L. E. Dickson : "An invariantive investigation of irreducible binary forms."

(9) Professor Arthur Ranum: "On the classification of systems of linear equations."

(10) Professor Arthur Ranum : "The osculating sphere of a developable surface."

(11) Professor Peter Field : "The theory of degenerate rational plane curves."

(12) Dr. J. E. Rowe : “Important covariant curves and a complete system of invariants of the rational quartic curve."

(13) Professor G. D. Birkhoff : "General theory of linear difference equations."

(14) Professor L. E. Dickson : "A fundamental system of invariants of the general modular linear group on $m$ variables."

(15) Professor S. E. Slocum : "A general formula for the shearing deflection of beams of arbitrary cross section, either variable or constant."

(16) Professor G. A. Miller : "Note on the solution of a system of linear equations."

(17) Professor G. A. Miller: "Some relations between substitution group properties and abstract groups."

(18) Professor JACOB Westuund: "On the relative discriminant of a certain Kummer field."

(19) Professor J. W. Bradshaw : "On a method of deriving infinite products from certain infinite series." 
(20) Mrs. Anna J. Pell : "Infinite systems of linear equations with unsymmetric systems of coefficients."

(21) Professor EDWARD KASNer : "Conformal invariants of curvilinear angles."

(22) Professor Florian CaJori : "Fourier's improvement of the Newton-Raphson method of approximation anticipated by Mourraille."

(23) Dr. LouIs Ingold : "Note on identities connecting certain integrals."

(24) President E. O. LovetT : "Generalizations of certain theorems concerning cases of collisions in the general problem of several bodies."

(25) Professor John Eiesland : "On minimal lines and surfaces in four-dimensional space."

(26) Professor John Eiesland : "Lie's line-sphere geometry from the standpoint of four-dimensional space."

(27) Professor E. D. RoE, JR. : "A generalized definition of limit."

(28) Professor E. D. Roe, JR. : "A new invariant function."

(29) Dr. H. H. MitchelL: "The subgroups of the hyperorthogonal group $H O\left(3, p^{2 k}\right) . "$

(30) Dr. H. BEck : "Ein Seitenstück zur Moebius'schen Geometrie der Kreisverwandtschaften."

Dr. Beck's paper was communicated to the Society through Professor White. In the absence of the authors the papers of Professors Dickson, Field, Slocum, Miller, Westlund, Mrs. Pell, Professors Kasner, Cajori, Dr. Ingold, President Lovett, Professor Roe, and Dr. Beck were read by title.

Professor Dickson's first paper appeared in full in the October Buluetin. Dr. Beck's paper will appear in the October number of the Transactions. Abstracts of the other papers follow below. The abstracts are numbered to correspond to the titles in the list above.

2. The second paper by Professor Dickson determines by two methods all the groups of binary transformations of determinant unity in the general Galois field. For the more interesting types of these groups there is constructed a fundamental system of invariants. The paper has been offered for publication in the American Journal of Mathematics.

3. The coefficients of a binary $m$-ic are elementary sym- 
metric functions of $m$ groups of two homogeneous variables; and those of a $p$-ary $m$-ic will be similar functions of $m$ groups of $p$ homogeneous variables when the latter form is the product of $m$ linear forms. This analogy between the binary form and the degenerate $p$-ary form is of fundamental importance in the study of the structure of non-degenerate $p$-ary forms. In the first part of Professor Glenn's paper general methods of resolving degenerate forms into linear and quadratic factors are developed, including the cases where the factors are multiple. It is found that this problem always reduces to the direct problem of solving algebraical equations in one unknown, and the necessary equations are developed. This process of resolving a degenerate form is then applied to a non-degenerate form, with the result that the latter is reduced to a certain canonical type. The following theorem is established for ternary forms : I et $f_{3 m}=a_{x}^{m}+b_{x}^{m-1} z+\cdots$ be the general ternary form, $a_{x}^{m}$ being the binary form of order $m$, and so on. Let $a_{x}^{m}$ be factored into real factors, and assume (1) that all of these factors are contained in $b_{x}^{m-1}$ to a multiplicity one less than in $a_{x}^{m}$. Then by the processes developed we can obtain

$$
f_{3 m}=f_{m}+\mu_{m-2}
$$

where $f_{m}$ is the product of real linear or quadratic factors or both. Assume (2) that the satellite $\mu_{m-2}$ (of order $m-2$ ) is a form of the same character as $f_{3 m}$ (in that (1) is satisfied); also that its satellite is of this character, and so on. Then

$$
f_{3 m}=f_{m}+f_{m-2}+f_{m-4}+\cdots+f_{2-\epsilon} \quad(\epsilon=1 \text { or } 2),
$$

where $f_{m-2 k_{i}}$ of order $m-2 k$ is degenerate into linear and quadratic factors.

4. The primary object of Mr. Carmichael's paper is to prove the existence of a fundamental system of simple analytic solutions of the system of $n$ linear homogeneous difference equations

$$
G_{i}(x+1)=x^{a} \sum_{j=1}^{n} a_{i j}(x) G_{i}(x) \quad(i=1, \cdots, n),
$$

where $\alpha$ is a constant and the $\alpha_{i j}(x)$ are single-valued and $\alpha_{i j}(x)=a_{i j}+\alpha_{i j}^{\prime} x^{-1}+\alpha_{i j}^{\prime \prime} x^{-2}+\cdots \quad(i, j=1, \cdots, n ;|x|>\mathrm{R})$, 
the constants $\alpha_{i j}$ being such that the roots $\rho_{i}$ of the equation

$$
\left|a_{i j}-\delta_{i j} \rho\right|=0, \quad \delta_{i j}=\left\{\begin{array}{l}
0 \text { if } i \neq j, \\
1 \text { if } i=j,
\end{array}\right.
$$

are in absolute value different from each other and from zero. The proof is obtained by means of a form of the method of successive approximation and a process of induction.

If we suppose that $(A)$ is solved for $G_{i}(x)$ in terms of $G_{i}(x+1),(i=1, \cdots, n)$, and that $x$ is then replaced by $x-1$, we may write

$$
G_{i}(x-1)=\sum_{j=1}^{n} A_{i j}(x-1) G_{i}(x) \quad(i=1, \cdots, n) .
$$

One result of the paper may now be stated as follows: $(A)$ has a fundamental system of solutions

$$
G_{1 j}(x), G_{2 j}(x), \cdots, G_{n j}(x) \quad(j=1, \cdots, n),
$$

each function of which is analytic throughout the finite plane except at sets of points $[\beta-r],(r=0,1,2, \cdots)$, where $[\beta]$ is the set of all singularities of the coefficients $A_{i j}(x)$. If $x$ approaches infinity along any line parallel to the positive real axis,

$$
\lim G_{i j}(x)\{\Gamma(x)\}^{-a} \rho_{j}^{-x} x^{-m_{j}}=\alpha_{i j},
$$

where $m_{i}$ and $\alpha_{i j}$ are constants depending on the constants $a_{i j}$ and easily determined.

An existence theorem for a single equation of the first order is already known and some work (quite incomplete and unsatisfactory) has been done for a single equation of the second order ; but, except for some recent important partial results obtained by means of the Laplace transformation, no further substantial progress in obtaining analytic solutions of difference equations seems to have been made.

5 . In the consideration of the projective geometry upon a quadric surface

$$
Q \equiv \sum_{\iota \kappa} a_{\iota \kappa} x_{\iota} x_{\kappa}=0, \quad a_{\iota \kappa}=a_{\kappa \iota}, \quad\left|a_{\iota \kappa}\right| \neq 0, \quad(\iota, \kappa=1,2,3,4),
$$

with special reference to the general correlation of space

$$
\Omega=\sum_{\iota \kappa} b_{\iota \kappa} x_{\iota} y_{\kappa}=0
$$


we are confronted with the question of determining a transformation

$$
H: \quad \rho x_{\iota}=\sum_{\kappa} h_{\iota \kappa} x_{\kappa}{ }^{\prime}, \quad\left|h_{\iota \kappa}\right| \neq 0,
$$

which when carried out simultaneously on the $x$ 's and $y$ 's will reduce $Q$ and $\Omega$ to more simple forms. Algebraically this is asking for the matrix $H$ such that, when we perform the operations

$$
H^{\prime} Q H=\bar{Q} \quad H^{\prime} \Omega H=\bar{\Omega},
$$

where $Q$ and $\Omega$ are the matrices of $Q$ and $\Omega$ respectively, the matrices $\bar{Q}$ and $\bar{\Omega}$ will be in a more simplified form.

Dr. Burgess determines a method of reduction applicable to matrices of order $n$ and gets the results for the cases $n=2,3$, 4 , and 5 .

6. Professor Klein has pointed out that the form problem of the Valentiner group $G_{360}$ in the plane would have the same import for the solution of the sextic that the icosahedral binary $G_{60}$ has had for the solution of the quintic. Professor Gordan has shown that only an "accessory" cubic equation is necessary for the purpose. He obtains one such equation as the characteristic equation of a connex $(1,1)$ whose coefficients are of degree six in the roots of the sextic. It is the object of Professor Coble's paper to show that a connex $(1,1)$ exists which is of similar use and whose coefficients are of degree four only in the roots. It is shown further that there is no accessory equation of lower degree in the roots. A resumé is then made of the necessary calculations, in order to estimate the difficulty of setting out explicit formulas in the algebraic part of the problem of the sextic.

7. It was shown by Bertini * that every involutorial birational transformation of the plane into itself can be reduced to one of four types, by transforming through appropriate quadratic inversions. Of these, three were already known, but the fourth, in which the lines of the plane go into curves of order 17 with eight basis points of multiplicity six, was new. In Professor Snyder's paper the equations of these transformations are given, and some properties determined. The problem is

* " Ricerche sulle trasformazioni univoche involutorie nel piano," Annali di Mat., ser. 2, vol. 8 (1877), pp. 244-286. 
closely associated with that of the determination of the ninth and tenth double points of a sextic having eight given double points.

8. In this paper Professor Dickson determines a fundamental system of two invariants of the group of all binary linear transformations in a finite field. By means of a three-term recursion formula the product $\pi_{m}$ of the irreducible forms of degree $m$ is expressed in terms of the fundamental invariants $\pi_{1}$ and $\pi_{2}$. Two forms are said to belong to the same class if one of them can be transformed into a constant multiple of the other by a linear transformation of determinant unity in the field. It is shown that there are as many classes of irreducible binary forms of degree $m$ as there are irreducible factors of $\pi_{m}$ when expressed as a function of a certain pair of invariants, the choice of which depends upon the evenness or oddness of the modulus. The cases $m<8$ are examined completely. The paper has been offered for publication in the Transactions.

9. In his first paper Professor Ranum proves that in all the solutions of a system of consistent linear equations the values of a particular set of unknowns will be connected by a linear relation if and only if the omission of the coefficients of these unknowns from the matrix of the system reduces the rank of the matrix. This result is a generalization of a recent theorem of Miller's, and leads to a classification of consistent systems of rank $r$ in $n$ unknowns with respect to the reduction in rank caused by the omission of the coefficients of one or more unknowns. The geometric significance of this classification is explained.

10. Of the eight spheres inscribed in a tetraedron formed by four neighboring tangent planes to a developable $D$, seven are infinitesimal ; the limiting position of the eighth is defined as an osculating sphere $S$ of $D . \quad S$ is dual to the well-known osculating sphere of a twisted curve. By means of this duality, which is even more complete in riemannian than in euclidean space, Professor Ranum derives some of the properties of $S$, among which are the following: $S$ is the limit of a sphere inscribed in two neighboring osculating cones of $D$. If $D^{\prime}$ is the edge of regression of $D$, and $R$ is the rectifying curve of $D^{\prime}$, then $R$ is the locus of the center of $S$. If $s$ is the arc of $D^{\prime}$ and if $r$ and 
$\rho$ are its radii of curvature and torsion, respectively, then the radius $r^{\prime}$ of $S$ is given by the formula

$$
\frac{1}{r^{\prime}}=\frac{d}{d s}\left(\frac{r}{\rho}\right) \text {. }
$$

At the point of contact of $D$ and $S$ their curve of intersection has in general a double point with distinct tangents. A necessary and sufficient condition that $S$ be a fixed sphere is that $D^{\prime}$ be a geodesic line on a cone.

11. A number of papers have been written (see Glenn, "The theory of degenerate plane curves," American Journal of Mathematics, January, 1910, where additional references are given) on the conditions that an equation must satisfy in order that the corresponding curve shall degenerate. Professor Field's paper considers the same problem for rational curves only. The method of attack consists in expressing the coordinates of a point on the curve rationally in terms of a parameter $\lambda$, so that the curve is defined by the equations

$$
\theta x=f_{1}(\lambda), \quad \theta y=f_{2}(\lambda), \quad \theta z=f_{3}(\lambda) .
$$

The coefficients are then varied so that $f_{1}(\lambda), f_{2}(\lambda), f_{3}(\lambda)$ have one or more factors which become equal. While the problem in this form is restricted, the results are correspondingly simple.

12. Dr. Rowe's paper, in four sections, consists of a treatment of the rational quartic curve in the plane. In Section I Salmon's work on the combinants of two binary quartics with some extension is used to find the equations of the covariant curves, especially those of the covariant conics, of the rational quartic. Among other results we obtain an independent analytic proof that the six flexes of the rational quartic are on a conic. Section II is devoted to finding a complete system of invariants for the rational quartic as combinant of the fundamental involution ; also, by a mere permutation or cyclic substitution of letters it is possible to derive important invariants of the rational quartic from the covariant curves of Section I. In the third section a new system of invariants is obtained by considering this curve as the section of the Steiner quartic surface by a plane, the invariants being expressible in terms of the symmetric functions 
of the coefficients of the cutting plane. In Section IV the connection between the two systems of invariants is established, and a method for passing from our invariants to those obtained in another way by Professor Coble in an article in the American Journal, volume 31 , number 2 , is given.

13. A linear difference system may be written in either of two equivalent ways,

$$
g_{i}(x)=\sum_{j=1}^{n} a_{i j}(x) g_{j}(x) \quad \text { or } \quad g_{i}(x-1)=\sum_{j=1}^{n} b_{i j}(x) g_{j}(x),
$$

where $i=1,2, \cdots, n$; and to each of these corresponds a complete set of formal solutions. By the use of these, Professor Birkhoff proves the existence of two complete sets of solutions, which are termed principal solutions in virtue of their remarkable properties. The only singularities of the first (second) set of solutions are poles which fall to the right (left) of and congruent to the poles of $a_{i j}(x)\left[b_{i j}(x)\right]$, and the asymptotic form at $x=\infty$ is completely specified except along the line of positive (negative) reals. The functions $a_{i j}(x)$ and $b_{i j}(x)$ are rational functions of $x$.

The relations between the two sets of principal solutions is found to be expressible in terms of certain rational functions of $e^{2 \pi i x}$. The asymptotic properties and these relations entirely characterize the two sets, as sets of principal solutions of a linear difference system with rational coefficients, and the number of characteristic constants is equal to the number of arbitrary constants in the given system. This leads to the formulation of the fundamental problem of linear difference equations. Finally it is shown that the solution may be made to depend on that of a like system in which the $a_{i j}(x)$ are linear in $x$; this system which generalizes the ordinary gamma function is treated in some detail.

14. In this paper Professor Dickson shows that the group $G_{m}$ of all linear homogeneous transformations $T$ on $m$ variables with coefficients in the Galois field of order $p^{n}$ has the fundamental system of invariants $L_{m}, Q_{m 1}, \cdots, Q_{m m-1}$, where

$$
\begin{array}{r}
L_{m}=[m-1, \cdots, 0], \quad Q_{m s}=[m, \cdots, s+1, s-1, \cdots, 0] / L_{m}, \\
{\left[e_{1}, \cdots, e_{m}\right]=\left|x_{i}^{p^{n e_{j}}}\right| \quad(i, j=1, \cdots, m) .}
\end{array}
$$


The latter determinant is multiplied by $|T|$ by a transformation $T$. Hence the $Q_{m s}$ are absolute invariants. The $m$ fundamental invariants are shown to be independent. In the proof that they form a fundamental system, use is made of the relations

$$
\begin{aligned}
& Q_{m 1}=Q_{m-11}\left(L_{m} / L_{m-1}\right)^{r}+L_{m-1}^{r p^{n}}, \\
& Q_{m s}=Q_{m-1 s}\left(L_{m} / L_{m-1}\right)^{r}+Q_{m-1 s-1}^{p^{n}} \quad(s>1),
\end{aligned}
$$

in which $Q_{m-1 s}$ is to be suppressed if $s=m-1$, while $r=p^{n}-1$. Expanding $L_{m}$ according to its last column, we may express $L_{m} / L_{m-1}$ in terms of $L_{m-1}, Q_{m-1 s}, x_{m}$. These relations enable us to express the $Q_{m s}$ as integral functions of the $x_{i}$. The proof that the $Q_{m s}$ are integral functions follows easily from their definition.

The solution of the " form problem" is discussed. Further applications are of the character indicated in paper No. 8. This paper has been offered for publication in the Transactions.

15. Professor Slocum's paper has for its main object the derivation of a general formula for the shearing deflection of a beam of arbitrary cross section, either variable or constant. The method used is based on Castigliano's application of the principle of least work. The result is embodied in the formula

where

$$
D=\int \frac{Q^{\prime}}{Q G} d x \int q^{2} d A,
$$

$D=$ shearing deflection at any fixed point $P$,

$Q=$ total shear on any section due to given loading,

$Q^{\prime}=$ total shear on any section due to concentrated unit load at $P$,

$q=$ unit shearing stress due to shear $Q$,

$G=$ modulus of shear,

$d A=$ element of area of cross section,

$d x=$ element of length of beam.

For a constant rectangular cross section it is shown that the formula reduces to

$$
D=\frac{6}{5} \int_{0}^{l} \frac{Q Q^{\prime}}{A G} d x
$$

and for a constant circular cross section it becomes 


$$
D=\frac{10}{9} \int_{0}^{l} \frac{Q Q^{\prime}}{A G} d x .
$$

The formula is then applied to the case where the moment of inertia varies linearly along the axis of the beam, as is approximately true of the ordinary commercial planer rail. Definite numerical applications of the formula are given in each instance, and the percentage of shearing deflection for a given ratio of length to depth is also discussed.

It is further shown that the method here used for determining the shearing deflection also leads to the formula deduced otherwise by Professor Fraenkel for flexural deflection, namely,

where

$$
D=\int \frac{M m}{E I} d x,
$$

$D=$ flexural deflection at any given point $P$,

$M=$ moment at any section due to given loading,

$m=$ moment at any section due to concentrated unit load at $P$,

$E=$ Young's modulus,

$I=$ static moment of inertia of cross section,

$d x=$ element of length of beam.

It is then shown that all the ordinary formulas for bending deflection, as deduced independently from the equation of the elastic curve in each case, are simply special cases of the above. The formula is then further applied to the case where the moment of inertia varies linearly along the axis of the beam, a case not heretofore treated. Numerical application of the result is then made to the type of planer rail mentioned above.

16. The main theorem proved by Professor Miller is as follows: The necessary and sufficient condition that a given unknown has the same value in all the possible solutions of a system of consistent linear equations is that the rank of the matrix of the system is diminished by omitting the coefficients of this unknown from this matrix. Hence each unknown in a system of consistent equations must either have the same value in every possible solution or it has an infinite number of different values in the totality of possible solutions. This evidently includes the theorem that a system of linear equations has zero, one, or an infinite number of solutions. The didactic impor- 
tance of the theorem was emphasized and it was pointed out that this theorem should have a place in every advanced course in algebra, as it rounds out the students' conception of a system of linear equations. It was also observed that the necessary and sufficient condition that a given unknown in a consistent system of linear equations may have the value zero in a solution of the system is that the rank of the augmented matrix of the system is reduced by the omission of the coefficients of this unknown whenever the rank of the matrix is reduced by this omission. Hence it results that the necessary and sufficient condition that an unknown may have a value different from zero in a consistent system is that the rank of the augmented matrix is not reduced by omitting the coefficients of this unknown.

17. If $H$ represents any subgroup of index $\rho$ under a group $G$, all the operators of $G$ may be arranged so as to give $\rho$ distinct sets as regards both right hand and left hand multiplication in the following forms:

$$
\begin{aligned}
G & =H+H S_{2}+H S_{3}+\cdots+H S_{\rho}, \\
& =H+S_{2} H+S_{3} H+\cdots+S_{\rho} H .
\end{aligned}
$$

The sets $H S_{a}, S_{\alpha} H(\alpha=2,3, \cdots, \rho)$ are called respectively right co-sets and left co-sets of $G$ as regards $H$ by Professor Miller, and he observed that every right co-set is composed of the inverses of all the operators of some left co-set, and vice versa. The necessary and sufficient condition that $H$ is invariant under a given right co-set is that this co-set is identical with some left co-set; and the necessary and sufficient condition that $H$ gives rise to a multiply transitive representation of $G$, or of one of its quotient groups whose order exceeds two, is that every right co-set as regards $H$ has at least one operator in common with every left co-set as regards $H$.

If $G$ involves $k n_{1} h$ operators which transform $H$ into a group having a subgroup exactly of index $n_{1}$ in common with $H$, then $K_{1}$ must have exactly $k$ transitive constituents of degree $n_{1}$ and vice versa, where $K_{1}$ is composed of all the substitutions which omit one letter in the transitive substitution group $K$ to which $H$ gives rise. If $S_{\alpha} H S_{\alpha}^{-1}$ has a subgroup of index $n_{1}$ in common with $H$ and if the largest invariant subgroup of $H$ contained in this common subgroup is of index $m_{1}$ under $H$, 
then the corresponding transitive constituent in $K_{1}$ is of order $m_{1}$ and of degree $n_{1}$. These theorems exhibit more completely the principle of duality as regards substitution groups and abstract groups.

18. In a paper presented by Professor Westlund at the February meeting the discriminant or fundamental number of the algebraic number field $k(\sqrt[p]{m})$ was determined. The object of the present paper is to show how the results thus obtained may be used for determining the relative discriminant of the Kummer field $k(\sqrt{m}, \rho)$, where $\rho$ is a primitive $p$ th root of unity, with respect to the field $k(\sqrt[p]{m})$.

19. Professor Bradshaw illustrates a method of deriving infinite products from certain infinite series by application to Gregory's series for $\frac{1}{4} \pi$. By adding to this series a series which itself vanishes, a succession of better and better convergent series is obtained. The first term in this succession of series approaches the form of the infinite product due to Wallis.

20. Properties analogous to those for biorthogonal systems of functions are obtained for biorthogonal systems of limited linear forms in infinitely many variables. If the biorthogonal system

is such that

$$
U_{i}(x)=\sum_{i=1}^{\infty} u_{i} x_{i}, \quad V_{i}(x)=\sum_{i=1}^{\infty} v_{i} x_{i}
$$

$$
L(\cdot) V_{i}(\cdot)=\sum_{i=1}^{\infty} l_{i} v_{i}
$$

is convergent for a limited linear form $L(x)$, then

$$
V_{i}(x)=T U_{i}(x),
$$

where $T$ transforms every limited linear form into a unique limited linear form, and

$$
L(\cdot) T L(\cdot) \geqq 0, \quad L_{1}(\cdot) T L_{2}(\cdot)=L_{2}(\cdot) T L_{1}(\cdot) .
$$

From these properties of $T$ Mrs. Pell shows that it has the form

$$
T L(x)=K(x \cdot) L(\cdot)
$$


where $K(x y)$ is a symmetric definite or semi-definite limited bilinear form. The converse theorem is also true.

If the unsymmetric limited bilinear form $L(x y)$ is such that there exists a symmetric definite limited bilinear form $K(y)$ such that

$$
M(x y)=L(x \cdot) K(\cdot y)
$$

is a symmetric bilinear form, then there exists a spectrum for the system of linear equations

$$
U_{i}=\lambda \sum_{j=1}^{\infty} k_{i j} u_{j}, \quad V_{i}=\lambda \sum_{j=1}^{\infty} k_{j i} v_{j}
$$

In particular, if $L(x y)$ is continuous, there exists at least one characteristic number. This condition is also necessary.

21. The angles considered by Professor Kasner are bounded by arcs of analytic curves, and the conformal transformations are required to be regular at the vertices. The paper presents the conditions for the equivalence of such angles. A necessary condition is, of course, that the angles have the same magnitude $\theta$. When $\theta / \pi$ is irrational this is also a sufficient condition. If, however, $\theta / \pi$ is rational, an infinite number of invariants of higher order exist. The case where $\theta$ is zero (horn angles) is discussed separately.

22. Fourier showed in publications of 1818 and 1831 that, if one and only one root lies between the real numbers $a$ and $b$, then the Newton-Raphson method of approximation to the roots of a numerical equation $f(x)=0$ yields with certainty successive approximations to this root if the first two derivatives of $f(x)$ do not vanish for values of $x$ lying in the interval between $a$ and $b$, and if, of those two limits, that one is chosen as the initial approximation which, when substituted for $x$, gives to $f(x)$ and $f^{\prime \prime}(x)$ the same sign. Professor Cajori showed in his paper that Fourier was anticipated 50 years in this by J. Raym. Mourraille, who published in 1768 at Marseilles a work entitled Traité de la résolution des équations en général. Mourraille arrived at somewhat simpler results by omitting Fourier's unnecessary condition that $f^{\prime}(x)$ shall not vanish. Fourier was also anticipated in part of his "Rule B."

23. Because of the general nature of the symbols and sym- 
bolic products of the symbolic invariant theory it is possible to apply the formulas of this theory in various special fields. In the present note Dr. Ingold interprets symbolic functions as ordinary functions of a real variable $x$ in a definite interval, say $0 \leqq x \leqq 1$, and symbolic products as integrals, in the interval $0 \ldots 1$, of ordinary products. With this interpretation all of the identities of the symbolic invariant theory become identities involving ordinary functions and integrals.

24. In the first section of President Lovett's paper, the fundamental formula of Lagrange's celebrated essay on the problem of three bodies is extended to the motion of a system under forces varying as any arbitrary function of the distance. This generalization is then specialized to the case of forces varying inversely as any positive power of the distance; for the latter problem the generalizations are constructed of the differential equations employed by Sundman * in characterizing the conditions for collision under the law of nature. The second section is occupied with the determination of the different cases of singularities in the motion of a system of three bodies, the forces varying as the masses and inversely as an arbitrary positive power of the distance. In the third section, the results of the first section are extended to the most general problem of $n$ bodies, by utilizing to this end Block's † extension of Sundman's formulas to the newtonian case of any number of bodies. Finally the fourth section studies certain of the conditions for simultaneous collision in the most general case of the $n$ body problem.

25. Professor Eiesland's first paper treats of minimal lines, congruences of minimal lines, and curved surfaces which on account of certain properties have been defined as minimal surfaces in space of four dimensions. If such a surface lies in a space of three dimensions, it becomes an ordinary minimal surface. A minimal surface of four-dimensional space is the middle surface belonging to the focal surface of a minimal (or isotropic) congruence of that space; it is generated by the $\infty^{2}$ minimal tangent spaces

$$
(s+t) x_{1}+i(t-s) x_{2}+(s t-1) x_{3}+i(s t+1) x_{4}+F(s t)=0 .
$$

* K. F. Sundman, "Recherches sur le problème des trois corps," Acta. Soc. Sc. Fen., vol. 34 (1907).

$\dagger$ H. Block, "Sur une classe de singularités dans le problème de $n$ corps," Medd. f. Lunds Astr. Obs., ser. 2, no. 6 (1909). 
26. Professor Eiesland's second paper deals with the projection of minimal configurations into ordinary space by means of the orthogonal projection

$$
X_{1}=x_{1}, \quad X_{2}=x_{2}, \quad X_{3}=x_{3}, \quad X_{4}=i R
$$

which transforms the vertex of a minimal cone in four-dimensional space into the center of the sphere of radius $R$ in ordinary space, while the minimal cone becomes the sphere, i. e., the cone

$$
\left(x_{1}-X_{1}\right)^{2}+\left(x_{2}-X_{2}\right)^{2}+\left(x_{3}-X_{3}\right)^{2}+\left(x_{4}-X_{4}\right)^{2}=0
$$

becomes when projected into the space $\left(x_{1}, x_{2}, x_{3}\right)$ (putting $\left.x_{4}=0\right)$ :

$$
\left(x_{1}-X\right)^{2}+\left(x_{2}-X_{2}\right)^{2}+\left(x_{3}-X_{3}\right)^{2}=R^{2} .
$$

It is shown that Lie's line-sphere geometry follows as an immediate corollary from this theory of projection.

27. The object of Professor Roe's first paper is to explain a logical point of view, without affecting any of the facts involved, from which a greater generalization of the definition of limit can be obtained than is the case with the ordinary definition. To secure this it is necessary to distinguish between infinity and an infinite variable, and to discuss the meaning of "number" for the case in hand. The definition of limit is then so phrased that not only is the usual definition logically included as a special case, but infinity is also included as a limit, as well as any complex numbers, and even the extension of the definition to higher complex numbers may be easily made.

28. The new invariant function discussed in Professor Roe's second paper is

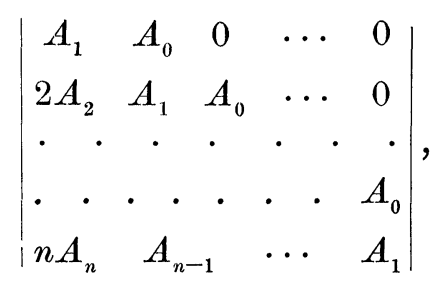

where $n>1, A_{k}=a_{0} b_{k}+a_{1} b_{k-1}+\cdots+a_{k} b_{0}$, and $b_{k}=\lambda^{k} / k !$.

The function is proved to be invariant, whatever the value of $\lambda$. 
It is used in the paper in finding general determinant values for the sums of the series

$$
\frac{1}{1^{2 n}}+\frac{1}{2^{2 n}}+\frac{1}{3^{2 n}}+\cdots, \quad \frac{1}{1^{2 n}}+\frac{1}{3^{2 n}}+\frac{1}{5^{2 n}}+\cdots
$$

29. A hyperorthogonal group $H O\left(3, p^{2 k}\right)$ which is representable as a collineation group in three variables, the coefficients being marks of the Galois field $G F\left(p^{2 k}\right)$, has an invariant of the form

$$
x_{1}^{p^{k+1}}+x_{2}^{p^{k}+1}+x_{3}^{p^{k}+1}=0 .
$$

It is of order $\left(p^{2 k}-p^{k}+1\right)\left(p^{k}+1\right)^{2} p^{3 k}\left(p^{k}-1\right) / d$, where $d=3$ or 1 , according as $p^{k_{i}}+1$ is or is not divisible by 3 .

Dr. Mitchell determines the subgroups of this group for $p$ an odd prime and $k$ a positive integer. The least number of letters on which the group is representable as a permutation group is $p^{3 k}+1$, except for $p^{k}=5$, in which case the least number is 50 .

F. N. CoLE, Secretary.

\section{THE PREPARATION OF COLLEGE AND UNIVER- SITY INSTRUCTORS IN MATHEMATICS.}

\section{PROVISIONAL REPORT OF THE AMERICAN SUBCOMMITTEE OF THE INTERNATIONAL COMMISSION ON THE TEACHING OF MATHEMATICS.*}

Table of Contents.

Preface

Page.

(1.............................................................. 78

I. Survey of past conditions......................................... 78

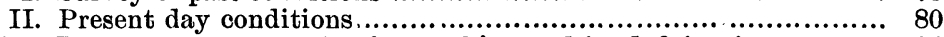

III. The present preparation for teaching and its deficiencies........... 84

IV. Supplements to the lecture system.................................. 87

V. Teachers' courses and teachers' colleges............................... 89

VI. The amount of graduate study ..................................... 92

VII. The supply and appointment of teachers.......................... 93

VIII. Concluding remarks.............................................. 99

* International Commission on the Teaching of Mathematics. Committee XIV -Graduate Work in Universities. Sub-committee 3-Preparation of Instructors for Colleges and Universities. Chairman, E. B. VAN VLECK, University of Wisconsin ; C. L. Bouton, Harvard University ; H. E. HAWKEs, Yale University ; G. D. OLDS, Amherst College ; H. E SLAUGHT, University of Chicago. This report was submitted to the American Commissioners in October, 1910. 